\title{
A influência dos stakeholders na elaboração de estratégia em pequenas empresas
}

\author{
The influence of stakeholders in the development of small business strategy
}

Carlos Alberto Freitas ${ }^{[a]}$, Sionesio Correa de Souza ${ }^{[b]}$

[a] Mestre em Administração pela Universidade do Vale do Itajaí (UNIVALI), professor da Universidade do Vale do Itajaí (UNIVALI), Itajaí, SC - Brasil, e-mail: carlosfreitas@univali.br

[b] Mestre em Administração pela Universidade do Vale do Itajaí (UNIVALI), professor do Instituto Federal de Santa Catarina (IFSC), Florianópolis, SC - Brasil, e-mail: sionesio@khronosnet.com.br

\section{Resumo}

O objetivo deste artigo foi analisar a relação entre as estratégias de influência dos Stakeholders no Processo de Elaboração de Estratégia em pequenas empresas. As hipóteses teóricas desta pesquisa partem da observação de que para uma organização obter vantagens competitivas é essencial perceber como as especificidades do ambiente externo influenciam suas decisões estratégicas. Os resultados apontaram como processo de elaboração predominante o Modo Empreendedor, seguido pelo Modo Incremental. Observou-se também que três Stakeholders no ambiente interno e quatro no ambiente externo poderiam empregar estratégias de influência. 0 estudo, de característica predominantemente quantitativa, foi realizado em 148 pequenas empresas entre 2005 e 2009. Realizaram-se associações por meio da utilização do coeficiente de contingência, além de análises de variância entre as abordagens propostas. Os resultados da associação entre as estratégias de influência que os Stakeholders podem empregar e os modos de elaboração de estratégia indicaram que existe associação, este resultado veio corroborar estudos anteriormente analisados. Outras associações foram realizadas, as análises de variância não apresentaram relação entre essas duas abordagens, entretanto, estatisticamente os resultados indicaram que não se deve refutar a hipótese de que existe associação entre as estratégias que os Stakeholders adotam e o processo de elaboração de estratégias. Outra associação foi entre a percepção das estratégias de influência que os Stakeholders podem empregar e o grau de formação dos pesquisados. Os resultados sugerem que quanto maior for o grau de formação do entrevistado mais ele percebe a influência do Stakeholders. Este resultado é relevante do ponto de vista teórico, visto que não se encontrou estudos avaliando esta relação. Esse estudo não apresenta evidências que possam afirmar que a adoção de um determinado modo de elaboração possa garantir uma vantagem competitiva. As informações obtidas nessas empresas parecem realçar a necessidade de que é preciso analisar mais variáveis quando se investiga pequenas empresas.

Palavras-chave: Stakeholders. Elaboração de estratégia. Pequena empresa. 


\section{Abstract}

The objective of this study was to analyze the relationship between influence strategies stakeholders in the process of developing strategies in small businesses. The hypotheses of this research is the observation that organization to gain competitive advantage is essential to understand how the specifics of the external environment influence their strategic decisions. The results showed as the process of drafting the predominant mode Entrepreneur, followed by an incremental fashion. It was also noted that stakeholders in three indoor and four in the external environment could employ strategies of influence. The study of predominantly quantitative trait was performed in 148 small businesses between 2005 and 2009. There were associations through the use of the contingency coefficient, and analysis of variance between the approaches. The results of the association between the influence strategies that stakeholders can use and ways of developing strategy indicated that an association exists, this result was corroborated previous studies analyzed. Other associations were made, analysis of variance showed no relationship between these two approaches, however, statistically the results indicated that one should not reject the hypothesis that an association exists between the strategies that the stakeholders and the process of adopting development strategies. Another association was between the perception of influence strategies that stakeholders can use and level of training of respondents. The results suggest that the greater the amount of training the respondent the more he realizes the influence of stakeholders. This result is relevant from a theoretical viewpoint, since we did not find studies evaluating this relationship. This study presents no evidence to say that the adoption of a particular mode of preparation can guarantee a competitive advantage. The information from these companies seem to emphasize the need that we have to consider more variables when investigating small businesses.

Keywords: Stakeholders. Strategy development. Small business.

\section{Introdução}

Por muito tempo pensar estratégia significava discutir o planejamento estratégico, entretanto isso se modificou já na década de 1970, quando Mintzberg (1973) escreveu que empreendedores realizavam suas estratégias de outras maneiras. Naquele período, Mintzberg (1973) chamou a atenção para a elaboração de estratégia em pequenas empresas, e desde então se percebe que tem se intensificado a atenção prestada à estratégia nesse tipo de negócio. Esta observação é confirmada pelo número de estudos relacionados à estratégica nessas organizações, como pode ser observado nos estudos de Gimenez et al. (1999); Cancellier (2001); Beaver e Price (2004); Aragon-Sánches e SánchesMarín (2005); Oliveira (2007); Hoffmann, Hoffmann e Cancellier (2009) e Fagundes e Gimenez (2009). Segundo Gimenez e Grave (1992), três abordagens principais têm direcionado as investigações em pequenas e médias empresas. A primeira seria a abordagem Econômica, que se refere ao número de funcionários, proporção de postos de trabalho gerados, participação no faturamento e valor adicionado, dentre outras desta natureza. A segunda é a Empreendedora, que investiga a criação de empresas, planejamento de novos negócios, capital de risco e franquias. E a última, a Administrativa, que se refere a pesquisas em administração em seus mais diversos aspectos, como planejamento; marketing; tomada de decisão; finanças; produção e recursos humanos. Sendo que as duas últimas têm sido mais frequentemente observadas.

Alguns trabalhos sobre pequenas empresas (GIMENEZ, 1998; HOFFMANN, 1998; GIMENEZ et al., 1999; CANCELLIER, 2001; ROSSETTO; ROSSETTO, 2001; BEAVER; PRICE, 2004; ARAGON-SÁNCHES; SÁNCHES-MARÍN, 2005; OLIVEIRA, 2007; HOFFMANN; HOFFMANN; CANCELLIER, 2009; FAGUNDES; GIMENEZ, 2009) focam sua preocupação sobre o processo de elaboração (o como?); as estratégias aplicadas (o que?); e as forças que influenciam diretamente sobre a escolha dessa estratégia (quem?). Esse estudo procura situar-se entre as pesquisas que evidenciaram de alguma forma ou individualmente estas abordagens, e com isso busca vincular exclusivamente o emprego das tipologias de processo de elaboração (o como?) e da teoria dos Stakeholders (quem?) aos trabalhos de estratégia em pequenas empresas. Tendo em vista esses aspectos, apresenta-se o seguinte problema de pesquisa: Qual a relação entre a percepção das estratégias de influência dos Stakeholders e o Processo de 
Elaboração de Estratégia que as pequenas empresas do estudo empregam?

\section{Fundamentação teórica}

\section{A influência dos stakeholders nas pequenas empresas}

Os questionamentos relativos ao ambiente competitivo surgem de diferenças no que se refere ao entendimento se o ambiente é um fenômeno realista, constituído por elementos visíveis, explícitos e de natureza concreta (DESS; BEARD, 1984; SHARFMAN; DEAN JUNIOR, 1991), ou nominalista, representando o caminho das informações do ambiente externo para empresas, validado pela crença e atenção dos gestores (WEICK, 1979). Ainda que não existam real e objetivamente dois ambientes (SCOTT, 2003; MACHADO-DA-SILVA; BARBOSA, 2002; MARCON; BANDEIRA-DE-MELLO; ALBERTON, 2008), a diferença conceitual tem se apresentado relevante para se avaliar nas análises organizacionais as influências institucionais que não são levadas em conta em estudos que partem de uma abordagem que objetiva exclusivamente as características materiais e econômicas do ambiente (PORTO et al., 2009). A literatura que trata dos stakeholders fundamenta que eles são importantes (FREEMAN, 1984) ou definidos como relevantes pelos gerentes quando avaliam seu poder, legitimidade e urgência (MITCHELL; AGLE; WOOD, 1997). Observa-se que esta influência pode ser direta ou indireta, e está ligada à dependência de recursos (ALDRICH; PFEFFER, 1976; PFEFFER; SALANCIK, 1978) entre a organização focal - aquela que está fornecendo ou obtendo recursos - e o próprio Stakeholders (FROOMAN, 1999), ou fundamentada na posição da organização na rede do Stakeholders (ROWLEY, 1997, p. 887-910).

Observa-se nos estudos de Freeman e Reed (1983) duas abordagens a respeito da teoria dos Stakeholders. Em sentido amplo eles apontam que Stakeholders são "qualquer grupo ou indivíduo, identificável e que possa afetar a consecução dos objetivos de uma organização ou que é afetado pela consecução dos objetivos de uma organização". Ressalta-se que esses determinados grupos podem ser os principais concorrentes, sindicatos, empregados e investidores, dentre outros. Em sentido restrito, Stakeholders seria "qualquer grupo ou indivíduo no qual a organização é dependente para a sua contínua sobrevivência".
Destaca-se que se encaixam nesta abordagem determinados fornecedores, instituições financeiras e acionistas, dentre outros.

De acordo com Stoner e Freeman (1985, p. 47), existem dois ambientes, "O ambiente de ação direta é formado por Stakeholders, indivíduos ou grupos que são diretamente ou indiretamente afetados pela perseguição aos seus objetivos por parte de uma organização". Conforme a abordagem de Stoner e Freeman (1985, p. 47) e Frooman (1999), eles pertencem a duas categorias de Stakeholders: os internos (1) formado por investidores, acionistas, proprietários, conselhos de administração e empregados; e os externos (2) - que englobam outros grupos, como concorrentes, sindicatos, fornecedores, clientes e determinadas agências governamentais. Segundo eles, a força empregada por um agente externo em um determinado componente da organização vai determinar se ele será ou não um Stakeholders. Já os elementos de ação indireta são aqueles do ambiente externo e que afetam a atmosfera onde a organização compete. Pode-se citar como elementos externos a política, a economia e a tecnologia. Aqueles autores sugerem que os elementos do ambiente geral podem ser classificados como: políticos, econômicos, sociais e tecnológicos e exercem influência em todas as organizações. 0 Quadro 1 apresenta a composição dos Stakeholders que influenciam de forma direta e indireta a organização.

De acordo com a tipologia de Frooman (1999), cada linha tem relação com a dependência, que pode alterar de alta e baixa, sendo que se avalia a variável dependência para observar se cada grupo é ou não dependente um do outro. A coluna vertical tem relação com a dependência da empresa com o Stakeholders e a horizontal com a dependência do Stakeholders com a empresa. Ele indica ainda quais estratégias os Stakeholders podem empregar para influenciar o comportamento da empresa, além dos elementos que podem esclarecer as estratégias escolhidas pelos Stakeholders.

Sintetizando o significado do Quadro 1, pode-se dizer que a estratégia direta é aquela em que o Stakeholders manipula o provimento de recursos; a estratégia indireta acontece quando o Stakeholders se associa a outro Stakeholders com o intuito de manipular de forma indireta o provimento de recursos, e assim influenciar as decisões que a empresa toma de acordo com seus interesses. A estratégia indireta de uso será empregada pelo Stakeholders quando ele 
for dependente (alta interdependência) da empresa. Quando a empresa é dependente (alta interdependência) do Stakeholders, esse utilizará a estratégia direta de retenção. Já a baixa interdependência resultará no emprego da estratégia indireta de retenção. Conforme Frooman (1999), o Stakeholders irá escolher uma estratégia direta de uso para influenciar a empresa se os dois forem dependentes um do outro.

\section{Processo de elaboração de estratégia} em pequenas empresas

Para Mintzberg (1973, p. 44-45), a estratégia nas pequenas empresas é conceituada como um padrão de comportamento, determinado pelas decisões tomadas diante da percepção do líder ante as mudanças do ambiente externo. Deve-se entender que o processo de formulação ou elaboração de estratégias depende fundamentalmente do intercâmbio de informações da organização com seu ambiente de

Quadro 1 - Universo de empregados convidados a responder a pesquisa

\begin{tabular}{|c|c|c|c|}
\hline \multirow{4}{*}{ 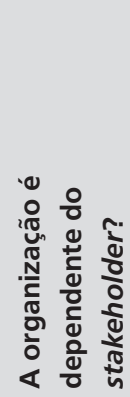 } & \multicolumn{3}{|c|}{ O stakeholder é dependente da organização? } \\
\hline & & Não & Sim \\
\hline & Não & $\begin{array}{l}\text { Indireta/recepção } \\
\text { (Baixa interdepen- } \\
\text { dência) }\end{array}$ & $\begin{array}{l}\text { Indireta/uso } \\
\text { (Poder da organi- } \\
\text { zação) }\end{array}$ \\
\hline & Sim & $\begin{array}{l}\text { Direta/retenção } \\
\text { (Poder do } \\
\text { stakeholder) }\end{array}$ & $\begin{array}{l}\text { Direta/uso } \\
\text { (Alta interdepen- } \\
\text { dência) }\end{array}$ \\
\hline
\end{tabular}

Fonte: Dados da pesquisa.

competição, assim como com seus recursos e capacidades internas. 0 conceito de elaboração de estratégia nas pequenas empresas passa pela não existência de um planejamento estratégico formal, pelo caráter improvisador, incremental e adaptativo das decisões dessas organizações, e que também se diferencia do rigor de uma estratégia planejada, por meio do planejamento estratégico normativo.

Percebe-se que a estratégia em pequenas empresas normalmente é desenvolvida pelo seu empreendedor, que geralmente é o proprietário. 0 capital desta organização é formado por um indivíduo ou por um pequeno grupo, e seu local de atuação é geralmente em um escritório no próprio empreendimento. São comumente organizações familiares e o dono normalmente é o responsável por tudo, sendo seu principal administrador. 0 poder e o processo de tomada de decisão caracteristicamente são centralizados nele, além de ser ele quem desempenha o principal papel no processo de formulação de estratégias (MINTZBERG, 1973; IDENBURG, 1993).

Para analisar o processo de elaboração é necessário entender como ele se desenvolve, mas para isso são necessários modelos ou modos que auxiliem neste entendimento. Assim, a partir de questionamentos como este, foi que Mintzberg (1973) desenvolveu seu trabalho, que chamou de modos de elaboração de estratégias. Um destes questionamentos refere à forma como as empresas tomam decisões importantes e como isto se relaciona ao processo de elaboração de estratégias na empresa. Ao procurar essas respostas, Mintzberg (1973) observou que o processo de elaboração de estratégias poderia desenvolver-se de três modos: o empreendedor, o adaptativo e o modo de planejamento.

Posteriormente, Quinn (1978, p. 7) contribuiu com o processo de elaboração de estratégias, ao defender que para se chegar à estratégia, o processo seria tipicamente fragmentado, evolucionário e largamente intuitivo. Para ele, o processo de elaboração das estratégias desenvolve-se em fases e cada uma sustenta-se nas anteriores. Nelas, a empresa pode seguir passo a passo, utilizando-se também de mudanças estratégicas devido à presença de forças dos ambientes interno e externo.

Outra contribuição ao estudo do processo de elaboração de estratégias vem de Idenburg (1993). Seu trabalho faz uma revisão teórica dos trabalhos de Mintzberg (1973) e Quinn (1978). A partir desta revisão, Idenburg (1993) aponta que seriam quatro e não três os modos de elaboração de estratégias: o planejamento racional, o incrementalismo lógico, a aprendizagem orientada e a estratégia emergente. Ao construir-se uma síntese dos textos seminais analisados, entende-se que o processo de elaboração de estratégias ocorre de maneira geral nas empresas e pode apresentar-se de cinco formas: a) 0 Planejamento Racional; b) 0 Incrementalismo Lógico; c) 0 Aprendizado Orientado; d) A Estratégia Emergente; e, por último, e) 0 Modo Empreendedor (MINTZBERG, 1973; QUINN, 1978; IDENBURG, 1993). Entretanto, neste estudo optou-se pela utilização do Planejamento Racional, do Incrementalismo lógico e 
do Modo empreendedor, por entender que eles podem explicar com maior nitidez o modo como as pequenas empresas constroem suas estratégias.

A literatura sobre estratégia na pequena empresa é influenciada por três abordagens: a Econômica, que foi a perspectiva dominante até 1984; a Empreendedora, que ganhou força com o reconhecimento da possível relevância da influência comportamental sobre o processo de elaboração e implantação de estratégia; e, por último, a abordagem Administrativa, que é composta pelos estudos diversos abordando inúmeras características da administração. Vários estudos relacionam o desempenho das pequenas empresas ao emprego de estratégias deliberadas, por meio do planejamento formal, e que contribuem para elevar as possibilidades de sucesso ou sobrevivência da empresa. Assim, parece não haver dúvidas no que tange os aspectos intuitivo, emergente e empreendedor do processo de elaboração de estratégias nas pequenas empresas, que além de se basear na experiência e aprendizado do indivíduo, busca também no ambiente externo angariar suporte para suas decisões estratégicas (GIMENEZ; RAMOS; FERREIRA, 2005, p. 859-871).

Destaca-se que o entendimento preponderante nas referências bibliográficas deste estudo observa a elaboração da estratégia como um processo que se desenvolve por meio de fases subsequentes, lógicas e indutivas, e que englobam um grupo de aspectos práticos fundamentados no contexto econômico e sem um planejamento formal para ajudar os gerentes na análise das escolhas estratégicas na hora de tomar as decisões nas empresas. Desta forma, surgem as dúvidas a respeito de como as empresas elaboram suas estratégias e como esse processo se desenvolve nas organizações, e, fundamentalmente, que relação tem com as estratégias genéricas que a organização adota e com as estratégias de influências dos Stakeholders.

Em seu estudo, Lima (2008) analisou o processo de elaboração de estratégia em pequenas empresas de móveis, verificando a influência que os Stakeholders têm no processo decisório e na elaboração das estratégias. Os resultados apontaram diferenças entre o tipo de influência exercida pelos Stakeholders internos e externos. Os internos normalmente exercem influência do tipo "efetiva e definidora" (significa que a empresa é dependente do Stakeholders, estratégia direta de uso). Segundo o autor, isso se deve em grande parte à proximidade desses Stakeholders com a formulação da estratégia, uma vez que eles estão em contato diário com as atividades da empresa e com os demais Stakeholders. Já nos externos predominam as influências de natureza "informativa/selecionadora e potencializadora" (significa que Empresa e Stakeholders são interdependentes, estratégia indireta de uso). Os resultados apontaram que os Stakeholders internos exercem influência mais decisiva no processo de elaboração, enquanto que os Stakeholders externos têm essa influência mais limitada.

\section{Metodologia da pesquisa}

Esse trabalho é um estudo de caso, com característica exploratória. A pesquisa de campo realizada foi predominantemente quantitativa, com enfoque descritivo, seguindo as orientações de Cervo e Bervian (1996). 0 universo da pesquisa compreendeu 148 micros e pequenas empresas comerciais do litoral norte catarinense que concordaram em participar da pesquisa (amostragem não aleatória por conveniência). Esta região é considerada um importante polo industrial e turístico, sendo uma das regiões economicamente mais importantes para o estado de Santa Catarina. A população aproximada de pequenas empresas formais do segmento de comércio e de serviços, distribuída entre os 19 municípios informados no questionário, é estimada em 17.500 empresas, que foram consideradas como universo da pesquisa. Foi realizado um pré-teste e foram efetuados os ajustes necessários. 0 estudo utilizou as informações de uma pesquisa realizada entre 2005 e 2009, e estes dados são inéditos. A amostra foi feita por conveniência, considerando que os participantes foram empresas que, ao serem contatadas, aceitaram tomar parte no estudo. A coleta de dados foi realizada de forma presencial diretamente na empresa. Foi realizada na maior parte dos casos com três pessoas em cada organização, com foco no perfil mais qualificado entre os respondentes: proprietários (59,46\%), gerentes $(29,73 \%)$, e outros $(10,81 \%)$. Sendo que mais de $73 \%$ dos respondentes estão há dois ou mais anos na empresa, e mais de $53 \%$ dos entrevistados têm ensino médio completo e outros $30 \%$ possuem curso superior.

0 questionário foi estruturado em três partes, a primeira com questões para definir a estratégia genérica predominante na empresa. A segunda parte investigou o processo de elaboração de estratégias e a terceira diz respeito aos Stakeholders e foi elaborada 
com o intuito de avaliar o nível de percepção (HAYES, 2003) dos entrevistados com relação à influência dos Stakeholders na empresa, e assim medir o nível de percepção da empresa com relação a eles. Seguindo a escala contínua de cinco pontos, sendo 5 para o grau mais alto e 1 para o mais baixo, segundo a classificação de Malhotra (2001).

Os Stakeholders selecionados foram aqueles que apresentaram mais de $50 \%$ das respostas do centro da escala para cima, ou seja, de 3 para cima. As estratégias de influência que os Stakeholders estão sujeitos a empregar foram identificadas no questionário, de acordo com as abreviações: ED - a empresa é dependente; SD - os Stakeholders são dependentes; e ESD a empresa e os Stakeholders são interdependentes. 0 tratamento se deu pela frequência absoluta, para Stakeholders, estratégia genérica, processo de elaboração e o coeficiente de contingência (C); para as medidas de associação entre Stakeholders, estratégia genérica e processo de elaboração. A seguir, analisou-se a associação entre as percepções do nível de influência dos Stakeholders com o grau de formação dos pesquisados. Para se chegar ao resultado calculou-se a média total de cada grau de formação, e assim determinou-se o nível de influência dos Stakeholders para cada grupo. A média de influência foi determinada por: ( $\sum$ pontos de cada Stakeholders)/16 - número de Stakeholders. Para determinar o índice de cada grau de formação utilizou-se a seguinte fórmula: $\left(\sum\right.$ pontuação total dos entrevistados por grau de formação) / N. Total de Stakeholders de cada grupo de entrevistados, e assim chegou-se a uma média total de cada grau de formação. Então foi empregada a matriz de correlação de Pearson.

\section{Resultados}

Os resultados da pesquisa dividem-se na determinação dos Stakeholders e seu grau de influência, para em seguida determinar o processo de elaboração de estratégia predominante. A partir dos resultados acima são apresentadas então as relações entre as duas abordagens.

\section{Determinação de stakeholders}

Os resultados da classificação dos Stakeholders vêm corroborar o que foi evidenciado por Stonner e Freeman (1985) e Hoffmann; Procopiak Filho e Rossetto (2008), que já apontavam que os Stakeholders do ambiente externo aparecem em maior número do que os do ambiente interno. Os resultados apontaram a existência de 13 Stakeholders externos, sendo que clientes, fornecedores e bancos e concorrentes são os mais evidentes (Tabela 1).

Percebe-se que as pequenas empresas dão muita importância a quatro Stakeholders externos: clientes $(88,5 \%)$, fornecedores $(70,9 \%)$, bancos $(43,6 \%)$ e concorrentes $(39,2 \%)$. Os resultados do nível de influência dos três primeiros podem ser evidenciados no trabalho de Sabino et al. (2005) e Procopiak Filho (2006), onde também se percebeu a elevada intensidade de influência desses três Stakeholders. Lima (2008) também confirma esse resultado em seu trabalho, ao apontar que o Stakeholders fornecedor é um dos que mais tem influência nas decisões estratégicas. Os demais Stakeholders apresentaram um percentual bem inferior em ambos os graus de importância. Hillman e Keim (2001) demonstraram que os Stakeholders externos possuem um elevado poder de influência nas decisões da empresa. Em contrapartida, podem fazer contribuições importantes, pois almejam o atendimento de seus interesses, e assim, podem proporcionar algumas vantagens e incentivos para empresa, o que parece explicar os resultados encontrados.

Na Tabela 2 percebe-se que todos os Stakeholders são importantes para a organização. Ambos se posicionaram com os maiores percentuais da população investigada, sendo grupo família com 80,4\%, funcionários com 76,4\% e por último os sócios, com $64,9 \%$, o que confirma a importância deste grupo de Stakeholders. Percebe-se nestes resultados que são idênticos aos observados por Sabino et al. (2005), Procopiak Filho (2006) e Lima (2008). Nota-se o elevado grau de importância imputado ao Stakeholders família e funcionários. Isso aparenta ser uma característica da pequena empresa, como visto neste trabalho, onde mais de $70 \%$ das empresas são gerenciadas pela família. Assim, a partir da importância de um Stakeholders para a empresa, é possível analisar as estratégias de influência que os Stakeholders do ambiente interno e do ambiente externo podem empregar.

Na Tabela 3 verifica-se o percentual de Stakeholders do ambiente externo que podem empregar estratégias de influência, $81,1 \%$ dos clientes podem utilizar essas estratégias, sendo que $65,5 \%$ podem adotar a 
estratégia direta de retenção. Ressalta-se a importância do grupo clientes no trabalho de Freeman e Liedtka (1997), onde o comportamento desse grupo na aquisição de produtos da empresa no longo prazo implica na reprodução desse comportamento, desta forma eles podem dar suas opiniões e sugestões no que se refere a produtos ou serviços. Também no trabalho de Procopiak Filho (2006), observa-se a predominância de uso por clientes da estratégia direta de retenção, da mesma forma que o demonstrado na Tabela 3.

A possibilidade de maior concentração de estratégias vem dos fornecedores, onde $87,2 \%$ deles podem empregá-las. Verificou-se no trabalho de Procopiak Filho (2006) que os fornecedores das empresas do setor de construção civil podiam adotar a estratégia indireta de uso, já neste estudo nota-se que a estratégia indireta de uso não é predominante entre as estratégias de influência que podiam ser usadas pelos fornecedores. Na questão referente aos fornecedores, pode-se observar na Tabela 3 que a predominância está na estratégia direta de retenção $(60,1 \%)$. Essa diferença na estratégia de influência

Tabela 1 - Distribuição do nível de influência dos stakeholders externos

\begin{tabular}{lccc}
\hline Stakeholders externos & $\%$ Nível 4 & $\%$ Nível 5 & $\%$ Total \\
\hline Clientes & $11,50 \%$ & $77,00 \%$ & $88,50 \%$ \\
Fornecedores & $19,60 \%$ & $51,40 \%$ & $70,90 \%$ \\
Bancos & $23,60 \%$ & $23,00 \%$ & $46,60 \%$ \\
Concorrentes & $29,10 \%$ & $10,10 \%$ & $39,20 \%$ \\
SPC & $19,60 \%$ & $12,80 \%$ & $32,40 \%$ \\
CDL & $16,20 \%$ & $12,20 \%$ & $28,40 \%$ \\
Associação comercial & $16,20 \%$ & $9,50 \%$ & $25,70 \%$ \\
Governo municipal & $15,50 \%$ & $9,50 \%$ & $25,00 \%$ \\
Governo estadual & $15,50 \%$ & $7,40 \%$ & $23,00 \%$ \\
Universidades & $9,50 \%$ & $10,10 \%$ & $19,60 \%$ \\
AMPE & $4,10 \%$ & $5,40 \%$ & $9,50 \%$ \\
SEBRAE & $4,10 \%$ & $4,70 \%$ & $8,80 \%$ \\
SENAC & $4,70 \%$ & $2,00 \%$ & $6,80 \%$ \\
\hline S & & & \\
\hline
\end{tabular}

Fonte: Dados da pesquisa. dos fornecedores entre os dois estudos talvez possa ser explicada pelo segmento de mercado que os dois estudos utilizaram. Enquanto Procopiak Filho (2006) analisou empresas da construção civil, esse estudo analisou pequenas empresas comercias. Essa divergência de resultados também pode estar relacionada ao número de fornecedores mono ou oligopolistas (cimento, ferro, elevadores e outros fornecedores) ligados à construção civil, enquanto que nas empresas comerciais não se observou a concentração de fornecedores poderosos. Em seguida vêm os bancos, com um percentual total de 50\%; desses, 37,8\% podem usar estratégia direta de retenção. Esse resultado aponta que aparentemente as pequenas empresas os utilizam como forma de financiamento para suas vendas (clientes) ou compras (fornecedores), ou talvez como investimento para ampliação do negócio. A seguir vêm os concorrentes, com um percentual total de 41,9\%; desses, 33,8\% podem exercer estratégia direta de uso. Isso ressalta uma elevada interdependência entre as pequenas empresas e os bancos, entretanto não se localizou estudos que avaliassem esta relação ou o Stakeholders bancos.

Tabela 2 - Distribuição do nível de influência dos stakeholders internos

\begin{tabular}{lccc}
\hline Stakeholders internos & $\%$ Nível 4 & $\%$ Nível 5 & $\%$ Total \\
\hline Família & $18,90 \%$ & $61,50 \%$ & $80,40 \%$ \\
Funcionários & $29,10 \%$ & $47,30 \%$ & $76,40 \%$ \\
Sócios & $10,10 \%$ & $54,70 \%$ & $64,90 \%$ \\
\hline
\end{tabular}

Fonte: Dados da pesquisa.

Tabela 3 - Distribuição das estratégias de influência dos stakeholders externos

(continua)

\begin{tabular}{lcccc}
\hline $\begin{array}{l}\text { Stakeholders } \\
\text { externos }\end{array}$ & $\%$ ED & $\%$ SD & $\%$ ESD & $\%$ Total \\
\hline Clientes & $81,10 \%$ & $2,70 \%$ & $14,90 \%$ & $98,60 \%$ \\
Fornecedores & $60,10 \%$ & $5,40 \%$ & $21,60 \%$ & $87,20 \%$ \\
Bancos & $37,80 \%$ & $1,40 \%$ & $10,80 \%$ & $50,00 \%$ \\
Concorrentes & $6,10 \%$ & $2,00 \%$ & $33,80 \%$ & $41,90 \%$ \\
SPC & $10,10 \%$ & $0,00 \%$ & $22,30 \%$ & $32,40 \%$ \\
\end{tabular}


Tabela 3 - Distribuição das estratégias de influência dos stakeholders externos

(conclusão)

\begin{tabular}{lcccc}
\hline $\begin{array}{l}\text { Stakeholders } \\
\text { externos }\end{array}$ & $\%$ ED & $\%$ SD & $\%$ ESD & $\%$ Total \\
\hline CDL & $10,80 \%$ & $0,70 \%$ & $18,90 \%$ & $30,40 \%$ \\
$\begin{array}{l}\text { Governo muni- } \\
\text { cipal }\end{array}$ & $9,50 \%$ & $0,00 \%$ & $15,50 \%$ & $25,00 \%$ \\
$\begin{array}{l}\text { Associação co- } \\
\text { mercial }\end{array}$ & $2,70 \%$ & $0,70 \%$ & $21,60 \%$ & $25,00 \%$ \\
$\begin{array}{l}\text { Governo estadual } \\
\text { Universidades }\end{array}$ & $6,80 \%$ & $0,00 \%$ & $16,20 \%$ & $23,00 \%$ \\
AMPE & $2,00 \%$ & $0,70 \%$ & $17,60 \%$ & $20,30 \%$ \\
SEBRAE & $2,00 \%$ & $0,00 \%$ & $7,40 \%$ & $9,50 \%$ \\
SENAC & $0,00 \%$ & $0,70 \%$ & $7,40 \%$ & $8,10 \%$ \\
\hline
\end{tabular}

Legenda: $E D=A$ empresa é dependente dos interesses dos Stakeholders;

SD $=$ Os Stakeholders são dependentes dos interesses da empresa;

ESD $=$ Empresa e os Stakeholders são interdependentes em razão dos seus interesses.

Fonte: Dados da pesquisa.

Nota-se na Tabela 4 as estratégias de influência que os Stakeholders do ambiente interno podem empregar. 0 grupo família, com $91,2 \%$ do total, aparenta ser o Stakeholders mais influente. Deste total, 52,7\% podem empregar a estratégia direta de retenção. Esse resultado já foi evidenciando anteriormente pelo nível de influência dos Stakeholders, e esta análise só vem confirmar aqueles resultados. Esse achado pode indicar o quanto esse Stakeholders pode influenciar o processo estratégico nessas pequenas empresas comerciais. Continuando, 89,2\% dos funcionários podem utilizar essas estratégias; e 50,0 \% deles podem empregar. Esse achado é relevante do ponto de vista teórico em razão de contrapor o estudo de Procopiak Filho (2006), que também analisou o poder de influência desses Stakeholders, pois naquele estudo apenas 3,4\% dos funcionários poderiam empregar a estratégia direta de retenção. Isso talvez possa encontrar explicação no porte das organizações analisadas nos dois estudos, pois as empresas de construção civil do estudo de Procopiak Filho (2006) são maiores se comparadas às desta pesquisa. Os sócios são outro grupo de Stakeholders influentes, onde $75 \%$ deles
Tabela 4 - Distribuição das estratégias de influência dos stakeholders internos

\begin{tabular}{lllll}
\hline $\begin{array}{l}\text { Stakeholders } \\
\text { internos }\end{array}$ & $\%$ ED & $\%$ SD & $\%$ ESD & $\%$ Total \\
\hline Família & $52,70 \%$ & $12,80 \%$ & $25,70 \%$ & $91,20 \%$ \\
Funcionários & $50,00 \%$ & $11,50 \%$ & $27,70 \%$ & $89,20 \%$ \\
Sócios & $49,30 \%$ & $4,70 \%$ & $20,90 \%$ & $75,00 \%$ \\
\hline
\end{tabular}

Fonte: Dados da pesquisa.

podem utilizar alguma estratégia de influência; sendo que $49,3 \%$ podem empregar a direta de retenção. Os resultados encontrados vêm corroborar os evidenciados por Sabino et al. (2005) e Lima (2008), pois a estratégia predominante no grupo sócio também foi a estratégia direta de retenção, onde 49,3\% das pequenas empresas os consideram um Stakeholders fundamental, com elevado nível de influência.

A partir dos resultados evidenciados nas análises, nota-se o elevado percentual de probabilidade do emprego de estratégias de influência por parte dos Stakeholders nas pequenas empresas comerciais pesquisadas.

\section{Determinação do processo de elaboração de estratégia}

Os resultados encontrados (Tabela 5) apontam como modo de elaboração de estratégia predominante o Empreendedor, ainda assim, muito próximo ao modo Incremental. 0 modo Empreendedor é citado por Mintzberg (1973) como característico das pequenas empresas. Uma dos aspectos marcantes deste processo é a forte presença do proprietário, onde normalmente há centralização de poder, e a transferência para a empresa de seus próprios objetivos pessoais (MINTZBERG, 1973, p. 44). Outros estudos identificaram os modos de elaboração de estratégia de forma individual e com outras abordagens, especificamente em pequenas empresas, como: Gimenez (1998), Cancellier (2001), Hoffmann (2002), Silva, Brandt e Costa (2003), Herrera, Abreu e Siqueira (2004), Sabino et al. (2005), Rathmann et al. (2007) e Hoffman, Hoffmann e Cancellier (2009).

Ao determinar o modo de elaboração adotado pelas pequenas empresas, verificou-se apenas 
uma exceção, o Planejamento Racional. As pequenas empresas apresentaram a predominância de dois modos de elaboração, aspecto previsto por Mintzberg (1973). As evidências aqui obtidas reforçam as observações de que nas pequenas empresas o Modo Empreendedor aparenta ser o mais presente (IDENBURG, 1993). Os resultados encontrados também corroboram o estudo de Oliveira (2007) e Hoffmann, Hoffmann e Cancellier (2009), que verificaram que as estratégias adotadas pelas pequenas empresas apresentavam características do Modo Empreendedor. Embora esse resultado já fosse esperado pelo autor em razão dos estudos avaliados na construção da fundamentação teórica, e que apresentavam o Modo Empreendedor como característico nessas empresas. Portanto, observa-se que o tamanho da empresa pode sim estar diretamente relacionado ao Modo Empreendedor.

Tabela 5 - Modo de elaboração de estratégias predominante

\begin{tabular}{lcc}
\hline $\begin{array}{l}\text { Processo de elaboração de } \\
\text { estratégia }\end{array}$ & N. empresas & Frequência \\
\hline INC - Incremental & 64 & $43,24 \%$ \\
EEM - Empreendedor & 69 & $46,62 \%$ \\
PR - Planejamento racional & 15 & $10,14 \%$ \\
Total & 148 & $100,00 \%$ \\
\hline
\end{tabular}

Fonte: Dados da pesquisa.

\section{Associação entre variáveis}

O nível de influência de cada Stakeholders pode variar de 1 a 5 . A pontuação máxima que cada respondente pode auferir aos stakeholders é de 80 pontos, ou seja, multiplica-se o número total de stakeholders (16) avaliados pela pontuação máxima (5) que cada stakeholders poderia atingir, e assim chega-se aos 80 pontos. Para calcular a média de influência dos stakeholders é somada a pontuação que cada respondente deu a cada um dos stakeholders, o resultado desta soma é divido pelo número de stakeholders (16). Para determinar o índice de cada grau de formação (Tabela 6) utilizou-se a seguinte fórmula, somou-se a pontuação total dos entrevistados por grau de formação e dividiu-se pelo número total de stakeholders de cada grupo de entrevistados, e assim chegou-se a uma média total de cada grau de formação.

Ao examinar a correlação entre o grau de formação do entrevistado e a sua percepção do nível de influência dos Stakeholders (Tabela 7), surge uma correlação positiva $(0,8585)$, apontando que quanto maior é o grau de formação do entrevistado, mais ele percebe a influência do Stakeholders na empresa. Esse resultado é relevante do ponto de vista teórico, pois não foram encontrados trabalhos que pudessem corroborar ou até mesmo descartar esse resultado, ainda que não se esteja falando de relação de causalidade.

Outra análise proposta nesse estudo foi verificar qualquer outra relação entre o processo de elaboração de estratégia e os Stakeholders através da ANOVA.

A Tabela de análise de variância (Tabela 8) decompõe a variância dos dados em dois componentes:

Tabela 6 - Relação entre as estratégias de influências dos stakeholders e grau de formação do entrevistado

\begin{tabular}{llll}
\hline & \multicolumn{3}{c}{ Stakeholders } \\
\cline { 2 - 4 } Grau de formação & $\begin{array}{c}\text { N. respon- } \\
\text { dentes }\end{array}$ & Frequência & $\begin{array}{c}\text { Nível de } \\
\text { influência }\end{array}$ \\
\hline $\begin{array}{l}1 \text { - Fundamental } \\
\text { (até } 8 \text { anos de } \\
\text { estudo) }\end{array}$ & 60 & $13,61 \%$ & 2,15 \\
$\begin{array}{l}\text { 2- Médio (até } 11 \\
\text { anos de estudo) }\end{array}$ & 236 & $53,51 \%$ & 2,29 \\
$\begin{array}{l}3 \text { - Superior } \\
\text { (até 16 anos de } \\
\text { estudo) }\end{array}$ & 130 & $29,48 \%$ & 2,62 \\
$\begin{array}{l}4 \text { - Pós-graduação } \\
\text { (mais de } 16 \text { anos } \\
\text { de estudo) }\end{array}$ & 15 & & \\
\hline \begin{tabular}{l} 
Total \\
\hline
\end{tabular} & 441 & $3,40 \%$ & 2,51 \\
\hline
\end{tabular}

Fonte: Dados da pesquisa.

Tabela 7 - Correlação entre o grau de formação e nível de influência dos stakeholders

\begin{tabular}{lc}
\hline & $\begin{array}{c}\text { Nível de influência dos } \\
\text { stakeholders }\end{array}$ \\
\hline Grau de formação & 0,8585
\end{tabular}

Fonte: Dados da pesquisa. 
Tabela 8 - Associação entre estratégias de influência dos stakeholders versus processo de elaboração de estratégia

\begin{tabular}{llcc}
\hline $\begin{array}{l}\text { Influência dos } \\
\text { stakeholders }\end{array}$ & \multicolumn{4}{c}{ Processo de elaboração de estratégia (\%) } \\
\hline Estratégias & \multicolumn{1}{c}{ INC } & EEM & PR \\
\hline Diretas/retenção & 67 & 67 & 33,5 \\
Indiretas/uso & 7,3 & 7,9 & 18,7 \\
Diretas/uso & 16,8 & 16,6 & 21,3 \\
Sem estratégia de & 9 & 8,5 & 26,5 \\
influência & 9 & 100 & 100 \\
\hline Total & 100 & & \\
\hline
\end{tabular}

Fonte: Dados da pesquisa.

entre os grupos (Processo de Elaboração e Stakeholders) e dentro do grupo (INC- Incremental; EEM - Empreendedor e PR - Planejamento Racional), e Estratégias de Influência: Diretas/Retenção; Indiretas/Uso; Diretas/Uso e sem estratégia de Influência. Assim, a ANOVA (Análise de Variância) analisa o comportamento da variável estratégia de influência dos Stakeholders nos diferentes modos de elaboração de estratégia (EEM/INC/PR). 0 p-valor da análise de variância resultou em 0,0691 (Tabela 9), apontado que deve-se refutar a suposição nula de que a variável estratégia de influência dos Stakeholders não depende dos diferentes modos de elaboração de estratégia nas pequenas empresas.

Percebe-se um nível de confiança de $90 \%$, apontando na ANOVA que a estratégia de influência dos Stakeholders é dependente do modo de elaboração de estratégia. Estatisticamente se pode afirmar que os modos de elaboração observados podem ser influenciados pela forma como as empresas percebem as estratégias de influência dos Stakeholders. Para confirmar o resultado, fez-se o gráfico de Box-andWisker (Figura 1), que confirma que a distribuição da variável estratégia de influência realmente depende do modo de elaboração observado. Para o modo Empreendedor é perceptível uma tendência de que a estratégia de influência esteja entre Diretas/Retenção e Diretas/Uso. No modo Incremental observa-se também a mesma tendência do modo Empreendedor, com a predominância das duas estratégias de influência. Por outro lado, no modo Planejamento Racional parece não haver associação alguma com as estratégias de influências dos Stakeholders. Nessa segunda análise, tem-se um p-valor $(0,149)$ que permite inferir com significância estatística da influência de uma variável sobre a outra. 0 gráfico confirma com a ANOVA, apontando que influências são essas e quais são os modos de elaboração que implicam.

Com este resultado infere-se que a ANOVA apontou a existência de uma significância estatística entre as variáveis, responde-se que as estratégias de influências podem ser elucidadas através dos modos de elaboração de estratégia. 0 gráfico apresenta exatamente as estratégias de influência dos Stakeholders, que influenciam para mais ou para menos os modos de elaboração de estratégias nas pequenas empresas comerciais. 0 modo de planejamento racional se apresentou mais neutro, observa-se também que a média tende para nível positivo, entretanto os outros quartis mostram que isso não tem muita relevância, talvez em razão da baixa população observada neste modo.

\section{Conclusões}

Este trabalho objetivou analisar a relação entre Stakeholders e o Processo de Elaboração de Estratégia em pequenas empresas comerciais dos municípios do litoral Norte Catarinense. Nos resultados da análise dos Stakeholders nas pequenas empresas, notou-se um elevado número de Stakeholders no ambiente externo em relação ao ambiente interno, resultado já esperado pelo pesquisador. Já no processo de elaboração, o modo Empreendedor identificado vem ratificar o previsto por Mintzberg (1973), assim como também o modo incremental proposto por Quinn (1978). Esse resultado corrobora o estudo de Hoffman (2002), onde o modo Empreendedor foi o mais presente nas pequenas empresas. Outro estudo que também corrobora os resultados encontrados é evidenciado, onde os estudos apontaram que a elaboração da estratégia na pequena empresa tem aspectos do modo Empreendedor. A relação entre o resultado encontrado nesse estudo e o observado nos demais trabalhos, que também identificaram o modo Empreendedor em pequenas empresas, indica que provavelmente esta variável reside no tamanho da empresa. Entretanto, apesar de o modo Empreendedor ser frequentemente encontrado em pequenas e médias empresas, já foi encontrado em empresas grandes (HOFFMANN, 1998). 
A influência dos stakeholders na elaboração de estratégia em pequenas empresas

Tabela 9 - Análise de variância para estratégia de influência do stakeholders e processo de elaboração de estratégia

\begin{tabular}{lllllll}
\hline Origem & Soma de quadrados & & G. L. & Quadrado médio & Razão F & p-Valor \\
\hline "Entre grupos" & 14,6793 & 3 & 4,78462 & 2,63 & 0,0691 \\
\hline Total & 122,5553 & 142 & & & \\
\hline
\end{tabular}

Fonte: Dados da pesquisa.

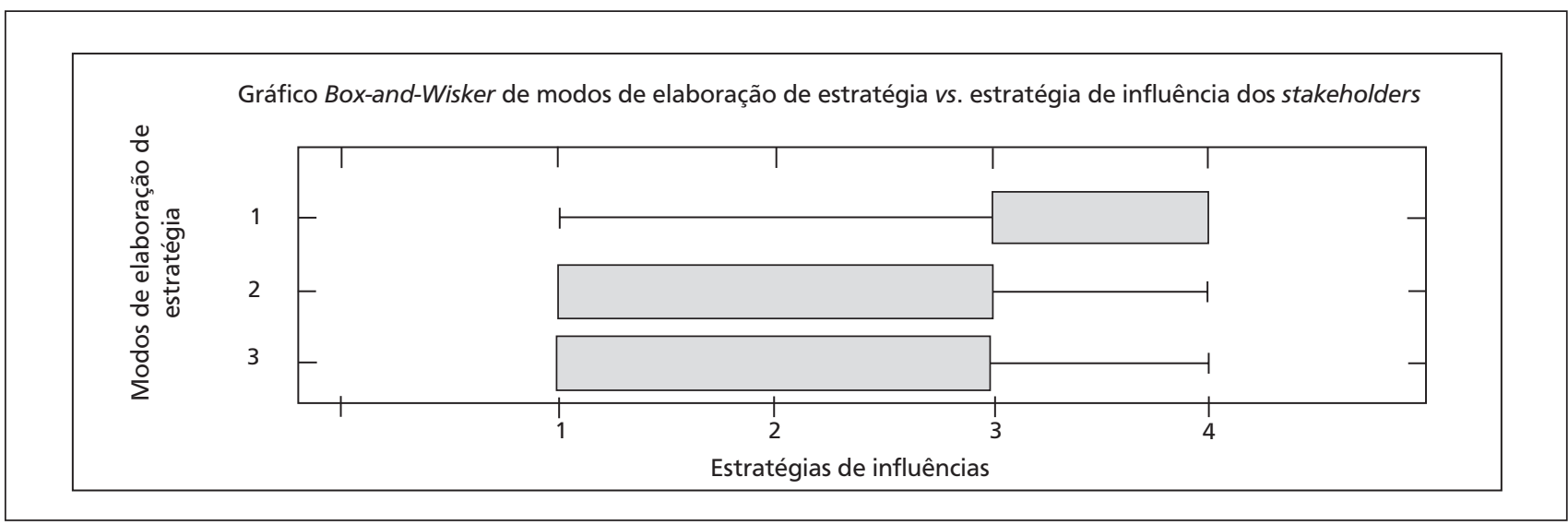

Figura 1 - Gráfico box-and-wisker de modos de elaboração versus estratégias de influência dos stakeholders Legenda: Modo de elaboração = 1 - planejamento racional; 2 - modo incremental; 3 - modo empreendedor.

Estratégias de Influência dos Stakeholders = 1 - diretas/retenção; 2 - indiretas/uso; 3 - diretas/uso; 4 - sem estratégia de influência. Fonte: Dados da pesquisa.

Outro resultado encontrado pode sugerir que quanto maior for o grau de formação do entrevistado, mais ele percebe a influência dos Stakeholders, indicando que o grau de formação dos indivíduos realmente influencia na forma como ele avalia a importância que o Stakeholders tem em relação à empresa. Esse resultado é relevante do ponto de vista teórico, pois até onde se conseguiu pesquisar, não se encontrou nenhum outro trabalho que fizesse menção a essa possível relação, embora ela faça sentido do ponto de vista teórico, e mesmo empírico. Outra observação situa-se em demonstrar que as empresas, ao estabelecerem suas estratégias, desprendem mais importância a determinados Stakeholders, em detrimento de outros. Como as empresas são dependentes de recursos, a atenção que elas promovem a determinado grupo de interesse está inteiramente conectada à necessidade básica de recursos que ela necessita para sua operação. 0 presente estudo corroborou o estudo de Stoner e Freeman (1985) e Frooman (1999) ao conseguir evidenciar através de seus resultados a validação das tipologias propostas por eles, e com isso também demonstrar a necessidade de um relacionamento mais próximo com esses Stakeholders, com o intuito de tornar mínimas as implicações que as suas estratégias de influência podem causar na tomada de decisão das pequenas empresas.

Sobre as recomendações e limitações existentes, existe a probabilidade de que se outros modelos teóricos ou outras questões fossem aceitos, diferentes resultados e novas conclusões poderiam ser encontrados. Por exemplo, o fato de escolher não analisar o Modo de Aprendizado Orientado e a Estratégia Emergente pode representar um viés de frequência absoluta adotada, mais que de sua inexistência. Além disso, não se teve acesso aos Stakeholders do ambiente externo, desta forma não se pode verificar se eles realmente empregam estratégias de influência identificadas nos resultados da investigação, como também já havia indicado Procopiak Filho (2006). 0 estudo ficou limitado aos aspectos quantitativos da pesquisa e apenas foram investigadas as empresas que aceitaram fazer parte do estudo. Entre as sugestões para 
futuras investigações, a mais provável seria elevar a ampliação do estudo nas cidades que já começaram a ser estudadas, assim como segmentar a pesquisa para pequenas empresas em outros segmentos de mercado, como indústrias e prestação de serviços. Como proposta para futuras pesquisas, pode-se aplicar um estudo de caráter mais qualitativo, com um reduzido número de casos, para ampliar a compreensão dos resultados de todo o grupo e também aplicar questionários nos Stakeholders envolvidos.

\section{Referências}

ALDRICH, H. E.; PFEFFER, J. Environments of organizations. Annual Review of Sociology, v. 2, p. 79-105, 1976.

ARAGON-SÁNCHES, A.; SÁNCHES-MARÍN, G. Strategic orientation, management characteristics, and performance: a study of Spanish SMEs. Journal of Small Business Management, v. 43, n. 3, p. 287-308, 2005.

BEAVER, G.; PRINCE, C. Management, strategy and policy in the UK small business sector: a critical review. Journal of Small Business and Enterprise Development, v. 11, n. 1, p. 34-49, 2004.

CANCELLIER, E. L. P. de L. A formulação de estratégias em pequenas empresas: um estudo na pequena indústria catarinense. In: ENCONTRO DA ASSOCIAÇÃO NACIONAL DE PÓS-GRADUAÇÃO E PESQUISA EM ADMINISTRAÇÃO, 25., 2001, Campinas. Anais... Campinas: EnANPAD, 2001.

CERVO, A. L.; BERVIAN, P. A. Metodologia científica. São Paulo: Prentice Hall, 1996.

DESS, G. G.; BEARD, D. W. Dimensions of organizational task environments. Administrative Science Quarterly, v. 29, n. 1, p. 52-73, 1984.

FAGUNDES F. M.; GIMENEZ, F. A. P. Ambiente, estratégia e desempenho em micro e pequenas empresas. REBRAE, v. 2, n. 2, p. 133-146, 2009.

FREEMAN, R. E.; REED, D. L. Stockholders and stakeholders: a new perspective on corporate governance. California Management Review, v. 25, n. 3, p. 88-92, 1983.

FREEMAN, R. E. Strategic management: a stakeholder approach. Boston: Pitman, 1984.

FREEMAN, R. E.; LIEDTKA, J. Stakeholders capitalism and the value chain. European Management Journal, v. 15, n. 3, p. 286-296, 1997.
FROOMAN, J. Stakeholders influence strategies. Academy of Management Review, v. 24, n. 2, p. 191-203, 1999.

GIMENEZ, F. A. P. Escolhas estratégicas e estilo cognitivo: um estudo com pequenas empresas. RAC, v. 2, n. 1, p. 2745, 1998.

GIMENEZ, F. A. P. et al. Estratégia em pequenas empresas: uma aplicação do modelo de Miles e Snow. RAC, v. 3, n. 2, p. 53-74, 1999.

GIMENEZ, F. A.; GRAVE, P. S. Discutindo a possibilidade de sistematizar o pensamento estratégico em pequenas empresas. Universidade Estadual de Londrina e Universidade Estadual de Maringá. In: ENCONTRO DA ASSOCIAÇÃO NACIONAL DOS CURSOS DE EM ADMINISTRAÇÃO, 8., 1992, Rio de Janeiro. Anais... Rio de Janeiro: ENAGRAD, 1992.

GIMENEZ, F. A. P.; RAMOS, S. C.; FERREIRA, J. M. O papel da análise da concorrência na formulação da estratégia em pequenas empresas. In: ENCONTRO DE ESTUDOS SOBRE EMPREENDEDORISMO E GESTÃO DE PEQUENAS EMPRESAS, 4., 2005, Curitiba. Anais... Curitiba: EGEPE, 2005.

HAYES, B. E. Medindo a satisfação do cliente: desenvolvimento e uso de questionários. Rio de Janeiro: Qualitymark, 2003.

HERRERA, V. É.; ABREU, A. de; SIQUEIRA, N. de A. Estratégia de diversificação e diferenciação numa pequena empresa de confeito de amendoim da capital nacional do alimento: estudo de caso da Yuppis Alimentos. In: SEMINÁRIO DE GESTÃO DE NEGÓCIOS, 1., 2004, Curitiba. Anais... Curitiba: FAE, 2004.

HILLMAN, A. J.; KEIM, G. D. Shareholder value, stakeholders management, and social issues: What's the botton line? Strategic Management Journal, v. 22, n. 2, p. 125139, 2001.

HOFFMANN, V. E. Processo de elaboração de estratégias organizacionais: evolução em uma empresa industrial. Revista Alcance, v. 1, n. 13, p. 75-78, 1998.

HOFFMANN, V. E. Comportamento estratégico em PME de primeira e segunda geração: um estudo de multicasos no Vale do Itajaí - SC. Revista Alcance, v. 4, n. 6, p. 79- 86, 2002. HOFFMANN, V. E.; PROCOPIAK FILHO, J. A.; ROSSETTO, C. R. As estratégias de influência dos stakeholders nas organizações da indústria da construção civil: setor de edificações em Balneário Camboriú - SC. Ambiente Construído, v. 8, n. 3, p. 21-35, 2008. 
HOFFMANN, R. A.; HOFFMANN, V. E.; CANCELLIER, E. L. P. de L. As estratégias da microempresa varejista e seus estágios de informatização. Revista de Administração Mackenzie, v. 10, n. 2, p. 110-134, 2009.

IDENBURG, P. J. Four styles of strategy development. Long Range Planning, v. 26, n. 6, p. 132-136, 1993.

LIMA, L. M. E. S. Influência dos stakeholders no processo estratégico de 2 pequenas empresas no ramo de móveis. 2008. 151 f. Dissertação (Mestrado em Administração) - Universidade Regional de Blumenau, Blumenau, 2008.

MACHADO-DA-SILVA, C. L.; BARBOSA, S. L. Estratégia, fatores de competitividade e contexto de referência das organizações: uma análise arquetípica. Revista de Administração Contemporânea, v. 6, n. 3, p. 7-32, 2002.

MALHOTRA, N. K. Pesquisa de marketing: uma orientação aplicada. Porto Alegre: Bookman, 2001.

MARCON, R.; BANDEIRA-DE-MELLO, R.; ALBERTON, A. Teoria instrumental dos stakeholders em ambientes turbulentos: uma verificação empírica utilizando doações políticas e sociais. Brazilian Business Review, v. 5, n. 3, p. 289-308, 2008.

MINTZBERG. H. Strategy-making in three modes. California Management Review, v. 16, n. 2, p. 44-53, 1973.

MITCHELL, R. K.; AGLE, B. R.; WOOD, D. J. Toward a theory of stakeholders identification and salience: defining that principle of who and what really counts. The Academy of Management Review, v. 22, n. 4, p. 853-886, 1997.

OLIVEIRA, I. C. J. de.; Processo de formação de estratégias em restaurantes: um estudo de múltiplos casos. 2007. 170 f. Dissertação (Mestrado em Administração) Universidade Federal de Pernambuco, Recife, 2007.

PFEFFER, J.; SALANCIK, G. R. The external control of organizations: a resource dependence perspective. New York: Harper \& Row, 1978.

PORTO, E. C. et al. Ambientes organizacionais: uma proposta de classificação com uso de munificência, dinamismo e complexidade. Revista de Administração e Contabilidade da Unisinos, v. 6, n. 2, p. 101-119, 2009.

PROCOPIAK FILHO, J. A. Estratégias e stakeholders em organizações da indústria da construção civil em Balneário Camboriú - SC. 2006. 166 f. Dissertação (Mestrado em Administração) - Universidade do Vale do Itajaí, Biguaçu, 2006.
QUINN, J. B. Strategic change: "logical incrementalism". Sloan Management Review, v. 20, n. 1, p. 7-21, 1978.

RATHMANN, R. et al. Fenadoce como estratégia de diferenciação e de vantagem competitiva dos doceiros de Pelotas. In: CONGRESSO DO INSTITUTO FRANCO-BRASILEIRO DE ADMINISTRAÇÃO DE EMPRESAS, 4., 2007, Porto Alegre. Anais... Porto Alegre: IFBAE, 2007.

ROSSETTO, C. R.; ROSSETTO, A. M. O processo de adaptação estratégica segundo o modelo de Tushman e Romanelli: um estudo de caso no setor de edificações. In: ENCONTRO NACIONAL DE ENGENHARIA DE PRODUÇÃO, 21., 2001, Salvador. Anais... Salvador: ENEGEP, 2001.

ROWLEY, T. J. Moving beyond dyadic ties: a network theory of stakeholders influences. The Academy of Management Review, v. 22, n. 4, p. 887-910, 1997.

SABINO, B. S. et al. A importância dos stakeholders na tomada de decisão das pousadas de Bombinhas. Revista Turismo, Visão e Ação, v. 7, n. 2, p. 341-354, 2005.

SCOTT, W. R. Organizations: rational, natural, and open systems. Upper Saddle River: Prentice Hall, 2003.

SHARFMAN, M. P.; DEAN JUNIOR, J. W. Conceptualizing and measuring the organizational environment: a multidimensional approach. Journal of Management, v. 17, n. 4, p. 701-710, 1991.

SILVA, J. F. da; BRANDT, E. A.; COSTA, L. S. de V. Truelo de tipologias estratégicas na arena das franquias de fast food no Brasil: Porter x Miles \& Snow x Mintzberg. In: ENCONTRO DA ASSOCIAÇÃO NACIONAL DE PÓS-GRADUAÇÃO E PESQUISA EM ADMINISTRAÇÃO, 27., 2003, Atibaia. Anais... Atibaia: EnANPAD, 2003.

STONER, J. A.; FREEMAN, R. E. Administração estratégica. Rio de Janeiro: PHB, 1985.

WEICK, K. The social psychology of organizing. 2nd. ed. Reading: Addison-Wesly, 1979.

Recebido: 23/09/2011

Received: 09/23/2011

Aprovado: 11/10/2011

Approved: 10/11/2011 